Communications in Physics, Vol.27, No. 1 (2017), pp. 45-53

DOI:10.15625/0868-3166/27/1/9241

\title{
AN ADVANCED LOGARITHMIC PHASE MASK FOR EXTENDING DEPTH OF FIELD OF HYBRID OPTICAL SYSTEMS
}

\author{
LE VAN NHU, PHAN NGUYEN NHUE, LE HOANG HAI ${ }^{\dagger}$ \\ Le Quy Don Technical University, \\ 236 Hoang Quoc Viet, Bac Tu Liem, Hanoi, Vietnam \\ ${ }^{\dagger} E$-mail: haiktq@gmail.com
}

Received 27 February 2017

Accepted for publication 31 March 2017

\begin{abstract}
In this paper, an advanced logarithmic phase mask is proposed and its performance is investigated. The essential performance characteristics of phase masks are shown, including the defocused modulation transfer functions (MTF), integral area of the MTF, Hilbert space angle, non-axial Strehl ratio, and decoded images. The results have demonstrated that our phase mask is highly beneficial to extend the depth of field of hybrid optical systems. The advantages of the proposed phase mask in comparison to some other masks are also pointed out.
\end{abstract}

Keywords: extended depth of field, hybrid imaging system, phase mask, wavefront coding.

Classification numbers: 42.30.Lr, 42.30.Va.

\section{INTRODUCTION}

Hybrid imaging systems, in which a phase mask is placed in the pupil plane to make the modulation transfer function (MTF) or the point spread function (PSF) less sensitive to defocus, can be used in many applications, such as aberrations reduction [1,2], thermal imaging [3,4], iris recognition $[5,6]$. In a hybrid imaging system, the incident wavefront is intentionally coded with a phase mask and the optically blurred images will be deblurred and decoded by a digital processing.

The essential part of hybrid imaging systems is the phase mask that is designed suitably to obtain invariant image quality with defocus. During the last years, a large number of researchers over the world have been keeping their eyes on the development of hybrid imaging system and many kinds of phase mask have been reported, such as cubic phase mask [1], logarithmic phase mask [7-10], exponential phase mask [11], sinusoidal phase mask [12,13], polynomial phase mask [14], tangent phase mask [15], rotation phase mask [16], and high-order phase mask [17]. Those phase masks enable one to extend the depth of field of optical systems to different ranges.

(C)2017 Vietnam Academy of Science and Technology 
Among the phase mask types, the logarithmic phase mask received much attention because of the better invariance of defocused MTFs $[8,9]$. The first odd-symmetric logarithmic phase mask $\left(f_{\text {Sherif_1 }}\right)$ was proposed by Sherif et al. in 2001 [7], and its improved version $\left(f_{\text {Improved } 1}\right)$ was presented in [8]. Both masks are based on combining a second order power function and a logarithmic function. The other masks, $\left(f_{\text {Sherif_2 }}\right)$ and $\left(f_{\text {Improved_2 } 2}\right)$, based on combining a fourth order power function with a logarithmic function, were reported in [9]. The phase functions of these four masks are written as follows,

$$
\begin{aligned}
f_{\text {Sherif_1 }}(x, y) & =\alpha \operatorname{sgn}(x) x^{2}(\log |x|+\beta)+\alpha \operatorname{sgn}(y) y^{2}(\log |y|+\beta) \\
f_{\text {Improved_1 }}(x, y) & =\alpha \operatorname{sgn}(x) x^{2} \log (|| x|+\beta|)+\alpha \operatorname{sgn}(y) y^{2} \log (|| y|+\beta|) \\
f_{\text {Sherif_2 }}(x, y) & =\alpha \operatorname{sgn}(x) x^{4}(\log |x|+\beta)+\alpha \operatorname{sgn}(y) y^{4}(\log |y|+\beta) \\
f_{\text {Improved_2 }}(x, y) & =\alpha \operatorname{sgn}(x) x^{4} \log (|| x|+\beta|)+\alpha \operatorname{sgn}(y) y^{4} \log (|| y|+\beta|)
\end{aligned}
$$

where $\alpha$ and $\beta$ are the parameters to control phase profile of the phase mask; $\operatorname{sgn}(x)$ represents the sign function which is defined as 1 for $x>0$ and -1 for $x<0$.

However, as shown in Refs. [8,9], the oscillation of the defocused MTFs at low spatial frequencies is an intrinsic drawback of the above phase masks which limits the acceptable defocus range. In this paper, we propose an advanced logarithmic phase mask to hold the defocused MTFs invariant over a long range of defocus. The superior performance of the phase mask is evaluated and demonstrated.

\section{PARAMETERS OF PHASE MASKS}

We propose a new phase mask with a phase function as below:

$$
f_{\mathrm{New}}(x, y)=\alpha x \log \left(1+\sqrt{\beta-x^{2}}\right)+\alpha y \log \left(1+\sqrt{\beta-y^{2}}\right),
$$

where $\beta \geq 1$.

Before evaluating performance of the phase mask, its parameters should be optimized at first. As we knew, an optimization procedure should satisfy two requirements. Firstly, the optical transfer function (OTF) should be kept invariant over a desired range of defocus. Secondly, the magnitude of MTF should be high enough for digital image processing. There are some metrics that can be used as criteria for the optimization procedure, such as Fisher information, root mean square error of the MTF or PSF. Here, we applied Fisher information for the optimization of phase mask parameters. As shown in Ref. [11], the merit function can be represented as follows,

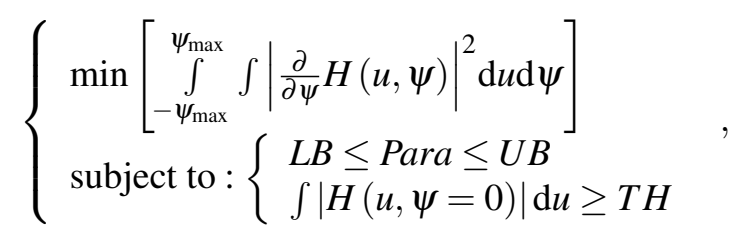

where $u$ is the normalized spatial frequency, $\psi$ denotes the defocus parameter and $\psi_{\max }$ is the maximum value of defocus parameter. $L B$ and $U B$ are the lower and upper bound of parameters of phase mask, respectively. Para is the parameters of the phase mask to be optimized. $H$ is the normalized OTF. $T H$ is a threshold to determine the acceptable minimum magnitude of MTF. Additionally, $T H$ is based on comparing performance of phase masks. 


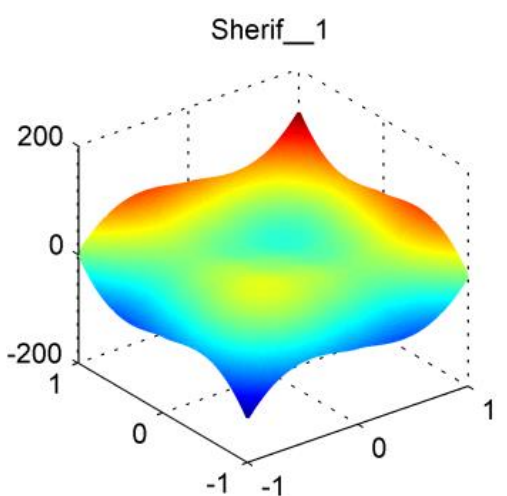

a)

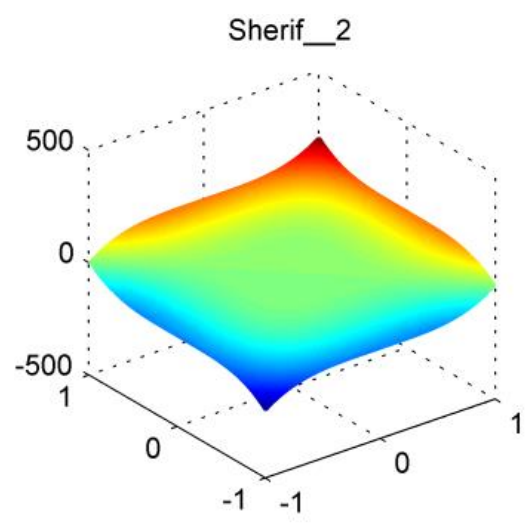

c)

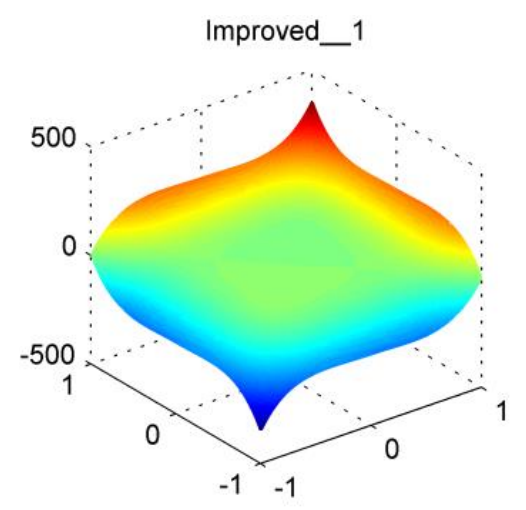

b)

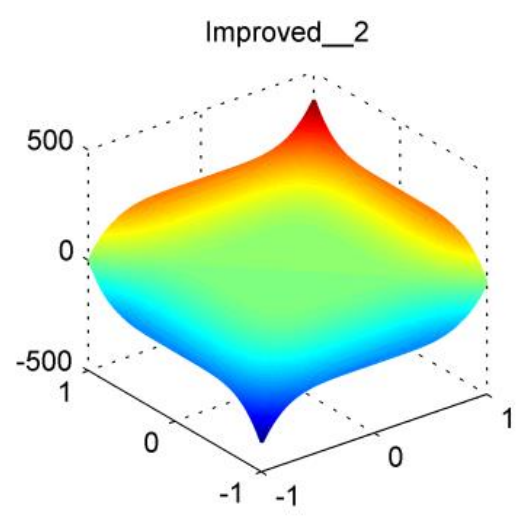

d)

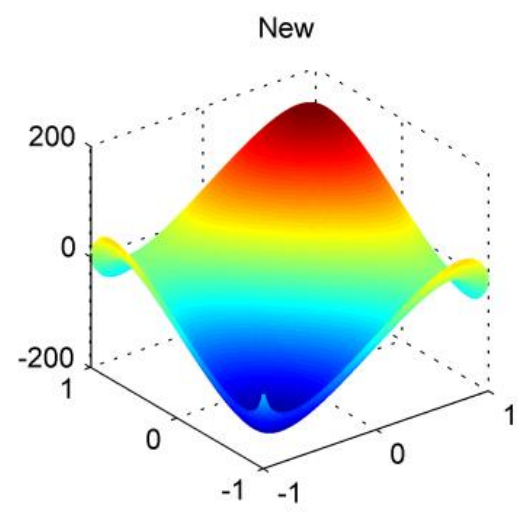

e)

Fig. 1. Phase profiles of five phase masks. 
Table 1. Optimum parameters of five logarithmic phase masks

\begin{tabular}{lrc}
\hline & $\alpha$ & $\beta$ \\
\hline \hline$f_{\text {Sherif_1 }}$ & 143.00 & 0.42 \\
$f_{\text {Improved_1 }}$ & -268.96 & -1.52 \\
$f_{\text {Sherif_2 }}$ & -23.13 & -4.44 \\
$f_{\text {Improved_2 }}$ & 275.38 & 0.97 \\
$f_{\text {New }}$ & 212.16 & 1.03 \\
\hline
\end{tabular}

In this paper, starting parameters are set to $T H=0.33$ and $\psi_{\max }=30$. By using optimization procedure as given in Eqs. (6), optimum parameters of the five phase masks are obtained as in Table 1 and their profiles are shown in Fig. 1 . As Fig. 1 indicates, the phase profiles of $f_{\text {Sherif_1 }}$,

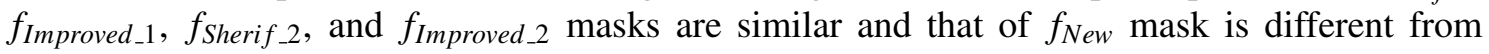
the others.

\section{PERFORMANCE EVALUATION OF PHASE MASKS}

With the data in Table 1, the defocused MTFs of five phase masks were computed and presented in Fig. 2. As shown in Fig. 2, the defocused MTFs of $f_{\text {Sherif } \_1}$ and $f_{\text {Improved_1 }}$ masks present the strong fluctuation at low spatial frequency part, whereas the MTFs of $f_{\text {Sherif } 2}$ and

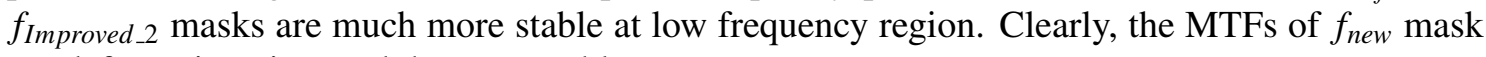
are defocus-invariant and the most stable.

Another way to consider stability of defocused MTFs is to evaluate the variation of integral area of the defocused MTF with respect to defocus [15]. The more stable integral area of the defocused MTF is, the more invariant phase mask to defocus will be. With the data in Table 1, the integral areas of the MTF defocused MTFs of five phase masks are represented in Fig. 3. As seen from Fig. 3, the integral area of defocused MTF of $f_{\text {Improved_1 }}$ mask is strongly changed with defocus, whereas $f_{\text {Improved_2 }}$ and $f_{\text {Sherif_. } 1}$ masks are more stable. Also, the $f_{\text {New }}$ mask is the most invariant to defocus.

Beside defocused MTFs, the most reliable way to evaluate similarity of defocused MTFs of a phase mask is to consider Hilbert space angle between the in-focus MTF and the out-focus MTF [15]. The Hilbert space angle between the focused MTF and the defocused MTF, $0 \leq \operatorname{thet} a(\psi) \leq$ $\pi / 2$, with defocus parameter $\psi$, can be written as,

$$
\theta(\psi)=\cos ^{-1}\left(\frac{\langle|H(u, 0)||H(u, \psi)|\rangle}{\||H(u, 0)||||||H(u, \psi)|||}\right)
$$

where the symbol $\langle\cdot\rangle$ implies the inner product and the symbol $\|\cdot\|$ denotes the norm. The smaller Hilbert space angle corresponds to the less variation in the MTF with respect to focus errors. 


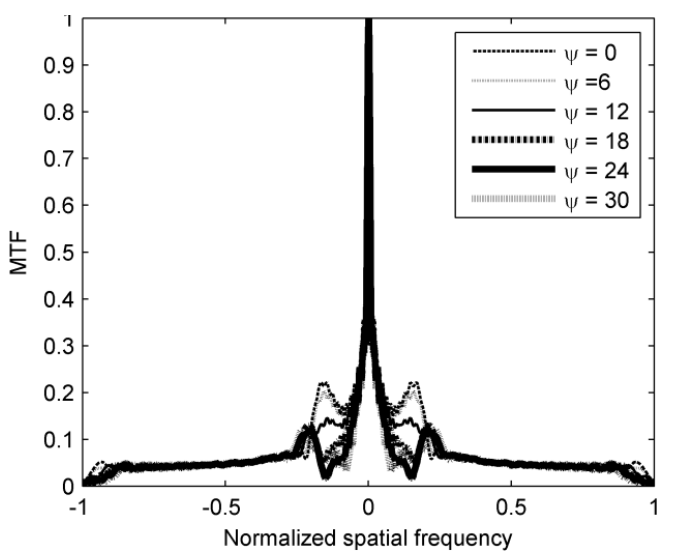

(a) $f_{\text {Sherif_1 }}$ mask

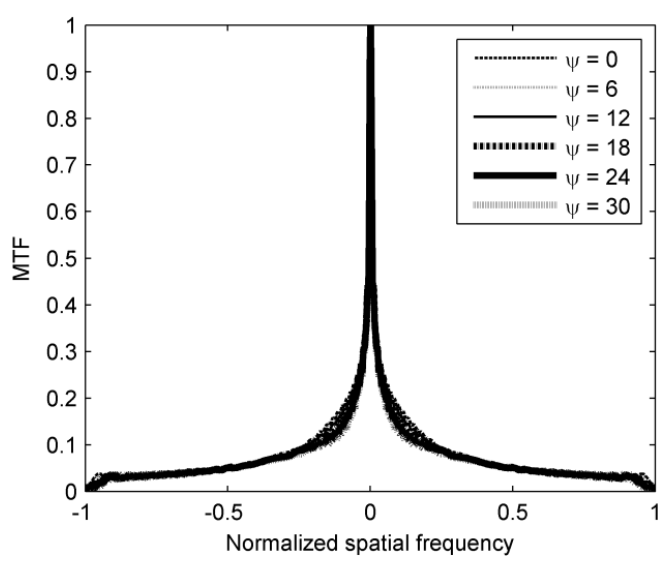

(c) $f_{\text {Sherif_2 }}$ mask

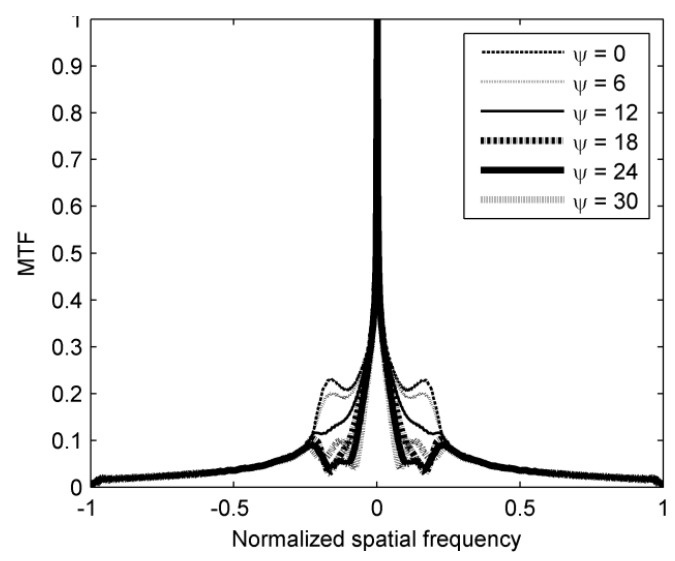

(b) $f_{\text {Improved_1 }}$ mask

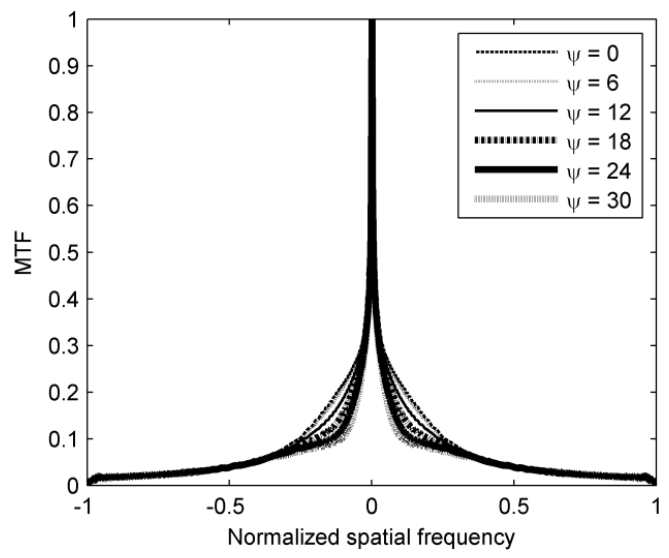

(d) $f_{\text {Improved_2 }}$ mask

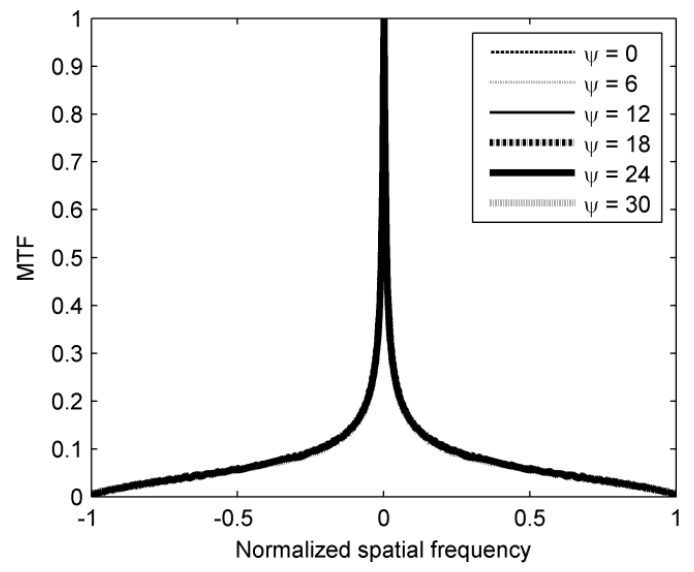

Fig. 2. Defocused MTFs of five phase masks. 


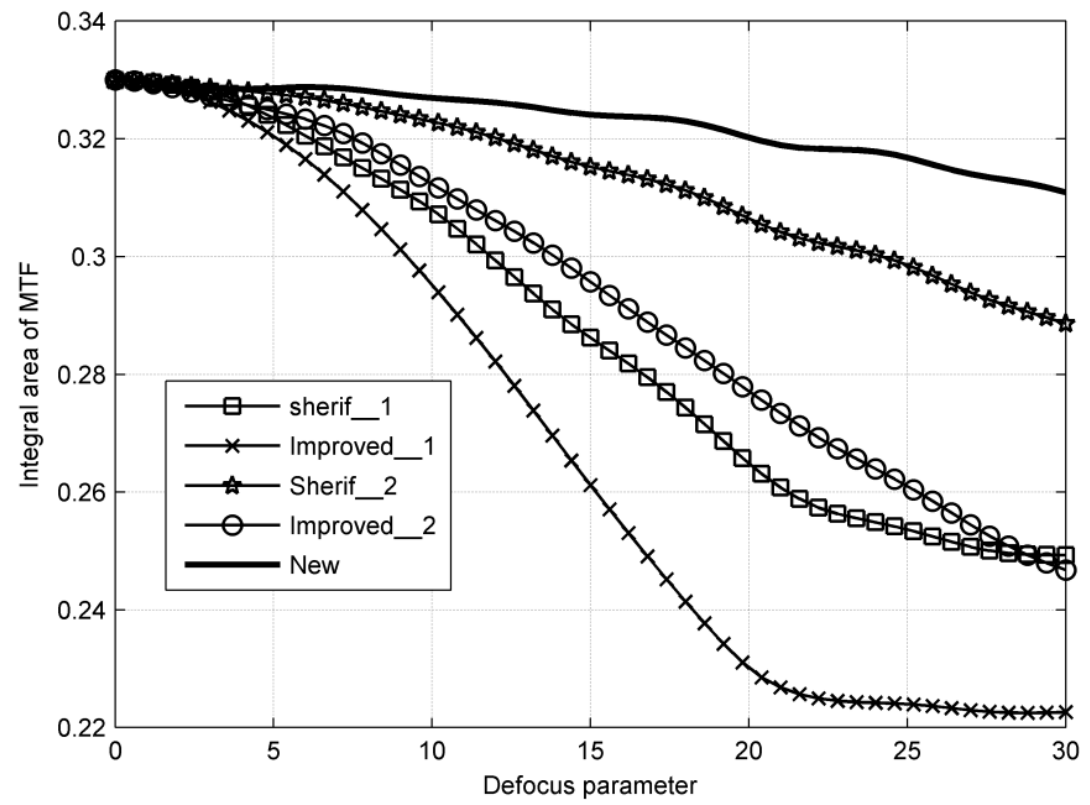

Fig. 3. Integral areas of the defocused MTF of five phase masks.

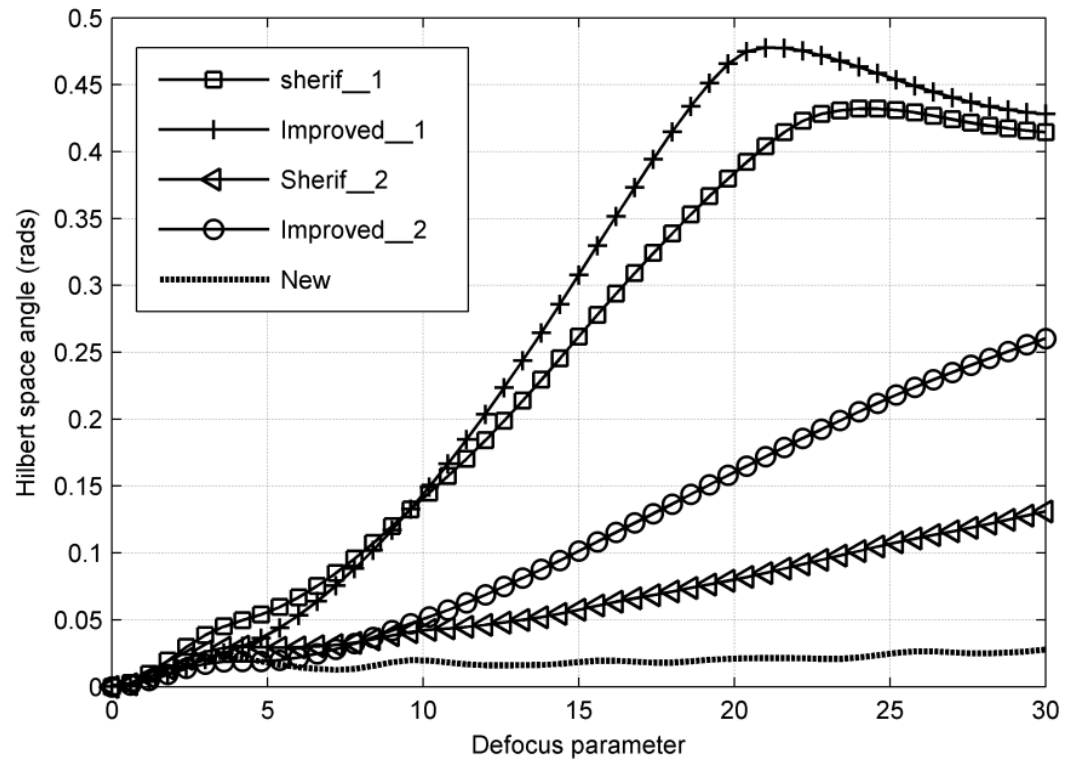

Fig. 4. Hilbert space angles of five phase masks. 
With the data in Table 1, Hilbert space angles of five phase masks are shown in Fig. 4. The Hilbert space angles of $f_{\text {Sherif_1 }}$ and $f_{\text {Improved_1 }}$ masks are the highest ones, the Hilbert space angles of $f_{\text {Sherif_2 }}$ and $f_{\text {Improved_2 }}$ masks are smaller than those of $f_{\text {Sherif_1 }}$ and $f_{\text {Improved_1 }}$ masks. Clearly, the Hilbert space angle of $f_{N e w}$ mask is the lowest one and it slowly changes with the increase of defocus. This means that the $f_{N e w}$ mask is nearly insensitive to defocus. This results in the better performance of the $f_{N e w}$ mask in extending the depth of field for hybrid imaging systems.

Next, another important approach to assess performance of phase masks is to consider the non-axial Strehl ratio of the PSF as defined in Ref. [18]. The variation of non-axial Strehl ratio of five phase masks is shown in Fig. 5. It is clear that the non-axial Strehl ratio of $f_{\text {Improved_1 }}$ mask varied strongly with defocus. Whereas, the $f_{\text {Sherif_1 } 1}$ and $f_{\text {Improved } 2}$ mask pair have the similar change. It is not difficult to see that the $f_{\text {New }}$ mask is the most invariant among the phase masks.

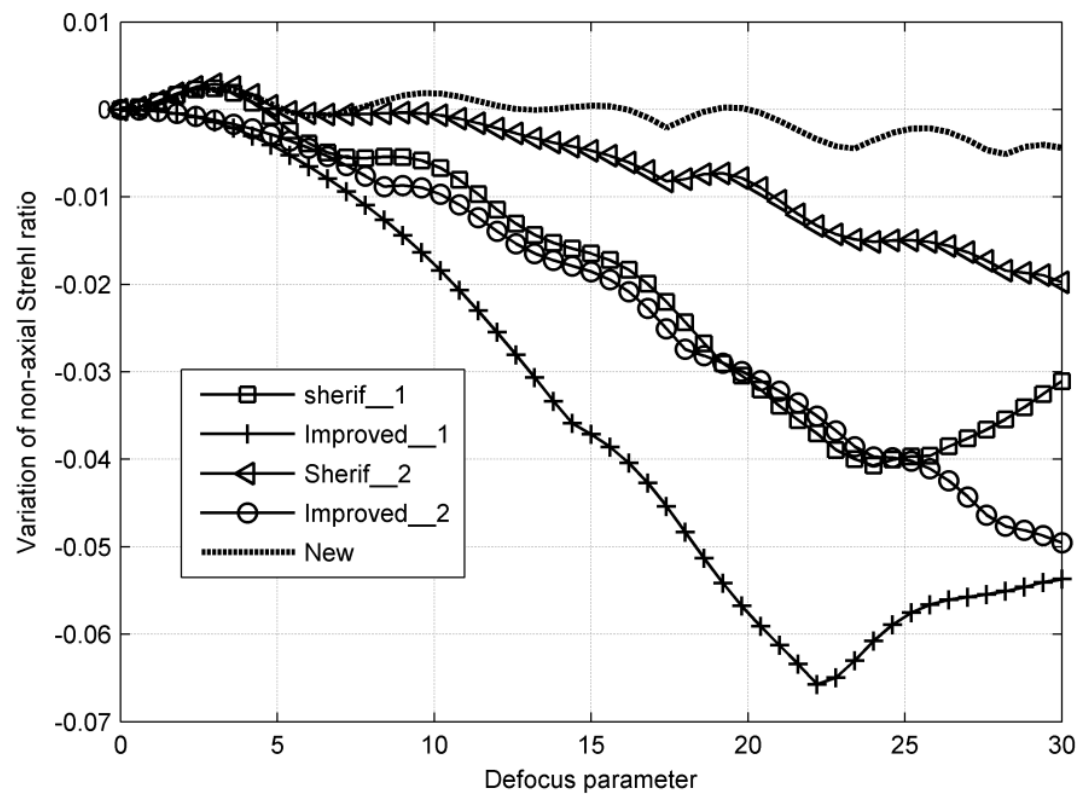

Fig. 5. Variations of the non-axial Strehl ratio of five phase masks.

Finally, a more direct way to evaluate the performance of phase masks is to examine the simulated images which can be obtained after a decoding [8]. Here, we use the samples of cameramen target. Fig. 6 shows restored images corresponding to five logarithmic phase masks at various defocus parameters of 0,15 , and 30. It can be seen from Fig. 6 that the $f_{\text {Sherif } 11}$ mask results in strong artifacts on the restored images, especially at large defocus parameter values, the $f_{\text {Sherif } 22}$ mask has less artifacts than the $f_{\text {Sherif_1 } 1}$ mask. For $f_{\text {Improved_1 }}$ and $f_{\text {Improved_2 }}$ masks, image artifacts on the restored images have been reduced obviously, but images are blurred at large defocus. It can be seen that the image artifacts on the decoded images of the $f_{N e w}$ phase mask are completely suppressed and the images still remain sharp at large defocus. This means that the $f_{N e w}$ mask enables to effectively extend the depth of field of hybrid imaging systems. 

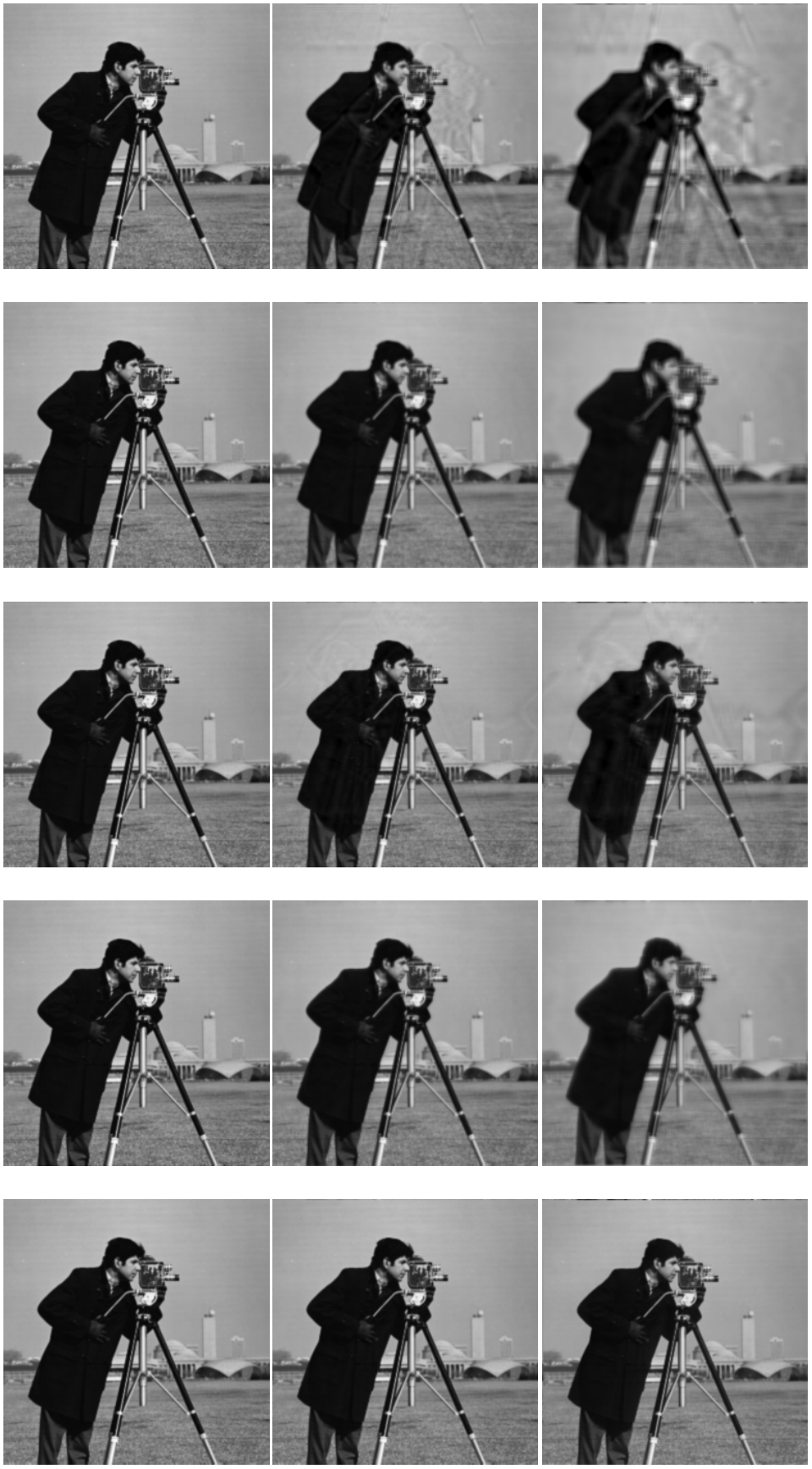

Fig. 6. Simulation images of cameraman target. (Rows from the top: $f_{\text {Sherif } \_1}, f_{\text {Improved } 11}$, $f_{\text {Sherif_2 }}, f_{\text {Improved_2 }}$, and $f_{\text {New }}$; Columns from the left: $\psi=0, \psi=15$, and $\psi=30$ ). 


\section{CONCLUSION}

In this paper, an odd-symmetric logarithmic phase mask has been proposed and examined. The investigated results of the defocused MTFs, integral area of the MTF, Hilbert space angle, non-axial Strehl ratio, and image restoration demonstrated that the proposed phase mask is very advantageous to keep the image quality invariant over a large range of defocus. The simulated images also proved that the proposed phase mask is highly effective to remove the image artifacts of the decoded images.

\section{REFERENCES}

[1] E. R. Dowski, Jr. and W. T. Cathey, Appl. Opt. 34 (11) (1995) 1859.

[2] S. Yuan, C. Preza, Opt. Express 19 (23) (2011) 23298.

[3] S. Chen, Z. Fan, Z. Xu, B. Zuo, S. Wang, H. Xiao, Opt. Lett. 36 (16) (2011) 3021.

[4] G. Muyo, A. Singh, M. Andersson, D. Huckridge, A. Wood, A. R. Harvey, Opt. Express 17 (23) (2009) 21118.

[5] R. Narayanswamy, P. E. X. Silveira, H. Setty, V. P. Pauca, J. van der Gracht, Proc. SPIE 5779 (2005) 41.

[6] D. S. Barwick, Appl. Opt. 47 (26) (2008) 4684.

[7] S. S. Sherif, E. R. Dowski, W. T. Cathey, Proc. SPIE 4471 (2001) 272.

[8] H. Zhao and Y. Li, Appl. Opt. 49 (2010) 229.

[9] H. Zhao and Y. Li, Opt. Lett. 35 (2010) 2630.

[10] H. Zhao, Q. Li and H. Feng, Opt. Lett. 33 (11) (2008) 1171.

[11] Q. Yang, L. Liu and J. Sun, Opt. Commun. 272 (2007) 56.

[12] H. Zhao and Y. Li, Opt. Lett. 35 (2010) 267.

[13] J. Wang, J. Bu, M. Wang, Y. Yang, X. C. Yuan, Opt. Lett. 37 (2012) 4534.

[14] N. Caron, Y. Sheng, Proc. SPIE 6832 (2007) 68321G.

[15] N. V. Le, Z. Fan, S. Chen, Opt. Lett. 39 (2014) 2171.

[16] F. Zhou, G. Li, H. Zhang and D. Wang, Opt. Lett. 34 (2009) 380.

[17] A. Sauceda, J. Ojeda-Castañeda, Opt. Lett. 29 (2004) 560.

[18] S. Chen, Z. Fan, H. Chang and Z. Xu, Appl. Opt. 50 (2011) 3337. 